\title{
Engineering Tuneable Light Harvesting Systems with Oligothiophene Donors and Mono- or Bis-Bodipy Acceptors
}

\author{
Samia Zrig, ${ }^{\dagger}$ Pauline Rémy, ${ }^{\dagger}$ Bruno Andrioletti, ${ }^{\dagger},{ }^{*}$ Eric Rose, ${ }^{\dagger}$ Inge Asselberghs ${ }^{\dagger}$ and \\ Koen Clays ${ }^{t, *}$
}

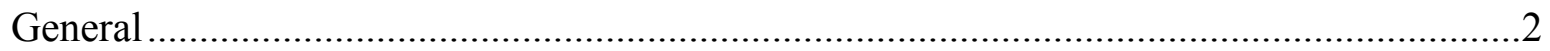

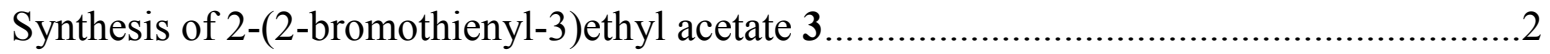

Synthesis of 3,3"'-di(2-acetoxyethyl)-5-formyl-2,2':5',2":5",2"'-quaterthiophene, 8 .............3

Synthesis of 5-BODIPY-3,3"'-di(2-acetoxyethyl)-2,2':5',2":5",2"'-quaterthiophene, 10 ........3

Normalized emission (blue, $\lambda_{\mathrm{exc}}=365 \mathrm{~nm}$ ) and excitation spectra (red, $\lambda_{\mathrm{em}}=460 \mathrm{~nm}$ ) for 64

Normalized emission (blue, $\lambda_{\mathrm{exc}}=605 \mathrm{~nm}$ ) and excitation spectra (red, $\lambda_{\mathrm{em}}=660 \mathrm{~nm}$ ) for 94

Concentration dependence spectrum for compound $\mathbf{1 0}\left(\mathrm{CHCl}_{3}\right)$....................................

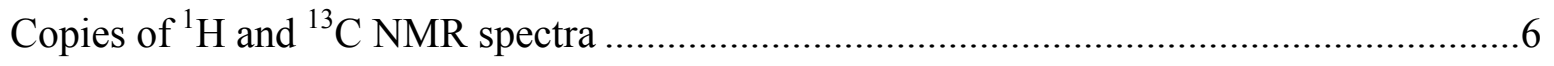




\section{General}

All reactions were carried out under an argon atmosphere unless otherwise noted. THF was distilled from sodium/benzophenone. DMF, DMSO, $\mathrm{NEt}_{3}$ and $\mathrm{CH}_{2} \mathrm{Cl}_{2}$ were distilled from calcium hydride. Commercial grade reagents and solvents were used without further purification unless otherwise stated. ${ }^{1} \mathrm{H}$ and ${ }^{13} \mathrm{C}$ NMR spectra were recorded at $400 \mathrm{MHz}$ and $100 \mathrm{MHz}$, respectively and were referenced to residual solvent. Products were purified by silica gel chromatography $\left(\mathrm{SiO}_{2} 15-40 \mu \mathrm{m}\right)$.

2-(2-bromothienyl-3)ethanol 2, 2,2'-bisthiophene 4 and the corresponding 2(tributylstannyl)-5-(5-(tributylstannyl)thiophen-2-yl)thiophene $\mathbf{5}$ were prepared according to literature procedure. ${ }^{1-3}$

\section{Synthesis of 2-(2-bromothienyl-3)ethyl acetate 3}

Pyridine $(1 \mathrm{~mL}, 12.3 \mathrm{mmol})$ was reacted with 2-(2-bromothienyl-3)ethanol 2 (2.02 $\mathrm{mg}, 9.82 \mathrm{mmol})$ in acetic anhydride $(7 \mathrm{~mL})$ for 2 hours. Excess of anhydride was removed in vacuo. The crude product was purified by silica gel column chromatography (dichloromethane) to give a colorless oil (2.35 g, $Y=97 \%)$.

${ }^{1} \mathrm{H}$ NMR $\left(\mathrm{CDCl}_{3}, 400 \mathrm{MHz}\right) \delta(\mathrm{ppm}) 7.22\left(\mathrm{~d}, 1 \mathrm{H}, J=5.68 \mathrm{~Hz}, \mathrm{H}_{5 \mathrm{Th}}\right), 6.83(\mathrm{~d}, 1 \mathrm{H}, J=$ $\left.5.68 \mathrm{~Hz}, \mathrm{H}_{4 \mathrm{Th}}\right), 4.24$ (t, 2H, $\left.J=6.82 \mathrm{~Hz}, \mathrm{H}_{1 \mathrm{Alk}}\right), 2.92$ (t, $\left.2 \mathrm{H}, J=6.82 \mathrm{~Hz}, \mathrm{H}_{2 \mathrm{Alk}}\right), 2.04$ $\left(\mathrm{s}, 3 \mathrm{H}, \mathrm{H}_{\mathrm{Me}}\right) ;{ }^{13} \mathrm{C} \mathrm{NMR}\left(\mathrm{CDCl}_{3}, 50 \mathrm{MHz}\right) \delta$ (ppm) 171.3, 137.5, 128.6, 126.0, 118.9, 63.6, 29.2, 21.3; MS $m / z$ calcd for $\mathrm{C}_{8} \mathrm{H}_{9} \mathrm{BrO}_{2} \mathrm{~S}$ 247.95. Found: $273[\mathrm{M}+\mathrm{Na}]^{+}$.

Yu, J.; Holdcroft, S. Macromolecules 2000, 33, 5073-5079.

Tamao, K.; Kodama, S.; Nakajima, I.; Kumada, M. Tetrahedron 1982, 38, 3347-3354.

Miller, L. L.; Yu, Y. J. Org. Chem. 1995, 60, 6813-6819. 
Synthesis of 3,3"'-di(2-acetoxyethyl)-5-formyl-2,2':5',2":5",2"'-quaterthiophene, 8

8 (25 mg; $0.047 \mathrm{mmol}$ ) was isolated as a byproduct from the formylation of 6 (160 $\mathrm{mg}, 0.318 \mathrm{mmol}$ ) in $15 \%$ yield.

${ }^{1} \mathrm{H} \mathrm{NMR}\left(\mathrm{CDCl}_{3}, 400 \mathrm{MHz}\right) \delta(\mathrm{ppm}) 9.80\left(\mathrm{~s}, 1 \mathrm{H}, \mathrm{H}_{\mathrm{CHO}}\right), 7.19(\mathrm{~d}, 2 \mathrm{H}, J=3.78 \mathrm{~Hz}$, $\left.\mathrm{H}_{\mathrm{Th}}\right), 7.10(\mathrm{~d}, 1 \mathrm{H}, J=4.04 \mathrm{~Hz}, \mathrm{H}), 7.07$ (d, 1H, $\left.J=3.78 \mathrm{~Hz}, \mathrm{H}\right), 7.00$ (d, 1H, $J=2.76$ $\mathrm{Hz}, \mathrm{H}), 6.99$ (d, 1H, $J=3.28 \mathrm{~Hz}, \mathrm{H}), 6.75$ (d, 1H, $J=3.56 \mathrm{~Hz}, \mathrm{H}), 4.33$ (t, 2H, $J=$ $\left.6.69 \mathrm{~Hz}, \mathrm{H}_{1 \mathrm{Alk}}\right), 4.28$ (t, $\left.2 \mathrm{H}, J=6.69 \mathrm{~Hz}, \mathrm{H}_{1 \mathrm{Alk}}\right), 3.14$ (t, 2H, $\left.J=6.69 \mathrm{~Hz}, \mathrm{H}_{2 \mathrm{Alk}}\right), 3.11$ (t, 2H, $\left.J=6.69 \mathrm{~Hz}, \mathrm{H}_{2 \mathrm{Alk}}\right), 2.07$ (s, 3H, $\left.\mathrm{H}_{\mathrm{Me}}\right), 2.04\left(\mathrm{~s}, 3 \mathrm{H}, \mathrm{H}_{\mathrm{Me}}\right) ;{ }^{13} \mathrm{C} \mathrm{NMR}\left(\mathrm{CDCl}_{3}\right.$, $100 \mathrm{MHz}) \delta(\mathrm{ppm}) 182.6,171.1,142.5,140.9,140.2,139.7,139.1,137.6,135.6$, 135.4, 134.8, 133.0, 128.9, 126.7, 125.3, 124.4, 124.3, 124.0, 64.5, 63.6, 29.8, 28.9, 21.2, 21.2; MS m/z: Calcd for $\mathrm{C}_{25} \mathrm{H}_{22} \mathrm{O}_{5} \mathrm{~S}_{4}$ : 530.0350, Found: 530.0395; UV-vis $\left(\mathrm{CH}_{2} \mathrm{Cl}_{2}\right) \lambda_{\max }(\varepsilon): 416(35160) ; \mathrm{mp}=102-104^{\circ} \mathrm{C}$.

\section{Synthesis of 5-BODIPY-3,3"'-di(2-acetoxyethyl)-2,2':5',2":5',2'"'- quaterthiophene, 10}

5-BODIPY-3,3"'-di(2-acetoxyethyl)-2,2':5',2":5",2"'-quaterthiophene $\mathbf{1 0}$ was synthesized from the monoformyl derivative $\mathbf{8}$ according to the experimental procedure described in the manuscript for the preparation of $\mathbf{1 0}$ in $45 \%$ overall yield.

${ }^{1} \mathrm{H}$ NMR $\left(\mathrm{CDCl}_{3}, 200 \mathrm{MHz}\right) \delta(\mathrm{ppm}) 7.93\left(\mathrm{~m}, 2 \mathrm{H}, \mathrm{H}_{\mathrm{pyr}}\right), 7.49\left(\mathrm{~s}, 1 \mathrm{H}, \mathrm{H}_{\mathrm{Th}}\right), 7.37$ (d, $\left.2 \mathrm{H}, J=4.16 \mathrm{~Hz}, \mathrm{H}_{\mathrm{pyr}}\right), 7.23\left(\mathrm{~d}, 1 \mathrm{H}, J=3.91 \mathrm{~Hz}, \mathrm{H}_{\mathrm{Th}}\right), 7.18\left(\mathrm{~d}, 1 \mathrm{H}, J=3.91 \mathrm{~Hz}, \mathrm{H}_{\mathrm{Th}}\right)$, $7.14\left(\mathrm{~d}, 1 \mathrm{H}, J=3.66 \mathrm{~Hz}, \mathrm{H}_{\mathrm{Th}}\right), 7.05$ (d, 2H, $\left.J=3.60 \mathrm{~Hz}, \mathrm{H}_{\text {pyr }}\right), 6.79$ (d, $1 \mathrm{H}, J=3.66$ $\left.\mathrm{Hz}, \mathrm{H}_{\mathrm{Th}}\right), 6.61\left(\mathrm{~d}, 2 \mathrm{H}, J=4.16 \mathrm{~Hz}, \mathrm{H}_{\mathrm{pyr}}\right), 4.41\left(\mathrm{t}, 2 \mathrm{H}, J=6.61 \mathrm{~Hz}, \mathrm{H}_{\mathrm{CH} 2}\right), 4.31(\mathrm{t}, 2 \mathrm{H}$, $\left.J=6.61 \mathrm{~Hz}, \mathrm{H}_{\mathrm{CH} 2}\right), 3.23\left(\mathrm{t}, 2 \mathrm{H}, J=6.71 \mathrm{~Hz}, \mathrm{H}_{\mathrm{CH} 2}\right), 3.14\left(\mathrm{t}, 2 \mathrm{H}, J=6.61 \mathrm{~Hz}, \mathrm{H}_{\mathrm{CH} 2}\right)$, $2.10\left(\mathrm{~s}, 3 \mathrm{H}, \mathrm{H}_{\mathrm{Me}}\right), 2.09$ (s, 3H, $\left.\mathrm{H}_{\mathrm{Me}}\right) ;{ }^{13} \mathrm{C} \mathrm{NMR}\left(\mathrm{CDCl}_{3}, 100 \mathrm{MHz}\right) \delta(\mathrm{ppm})$ 171.3, $171.2,144.0,140.3,139.7,139.6,139.0,137.7,136.7,136.1,135.8,135.0,134.3$, 133.0, 132.8, 132.3, 131.4, 128.7, 126.9, 125.4, 124.6, 124.4, 124.2, 118.8, 64.7, 63.9, 29.9, 29.0, 21.3; HRMS $m / z$ : Calcd for $\mathrm{C}_{33} \mathrm{H}_{27} \mathrm{BF}_{2} \mathrm{~N}_{2} \mathrm{O}_{4} \mathrm{~S}_{4}$ : 692.0915. Found: 
$673.0827[\mathrm{M}-\mathrm{F}]^{+}$; UV-vis $\left(\mathrm{CH}_{2} \mathrm{Cl}_{2}\right) \lambda_{\max }(\varepsilon): 384$ (25530), 514 (41620); sublimes > $130^{\circ} \mathrm{C}$.

Normalized emission (blue, $\lambda_{\text {exc }}=365 \mathrm{~nm}$ ) and excitation spectra (red, $\lambda_{\text {em }}=460$ nm) for 6

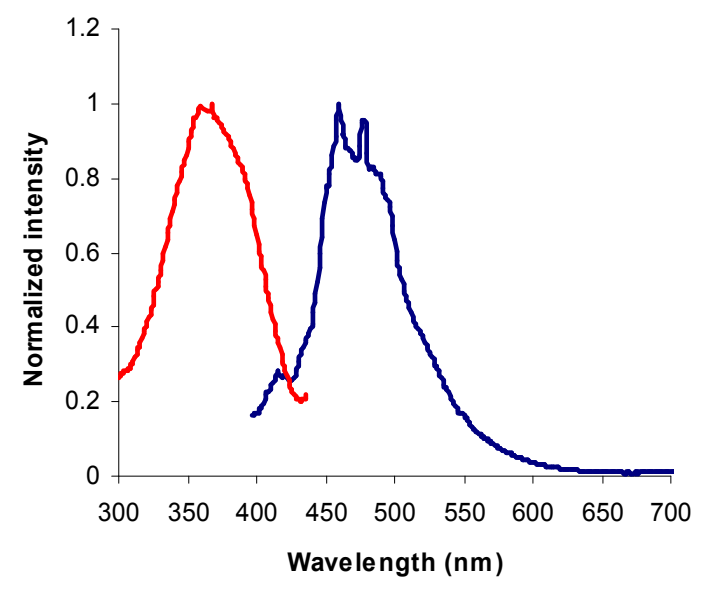

Normalized emission (blue, $\lambda_{\text {exc }}=605 \mathrm{~nm}$ ) and excitation spectra (red, $\lambda_{\text {em }}=660$ nm) for 9

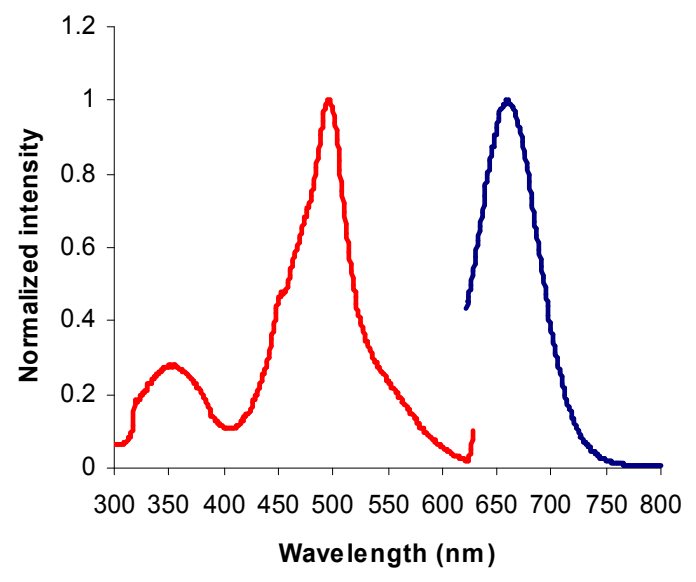


Concentration dependence spectrum for compound $10\left(\mathrm{CHCl}_{3}\right)$

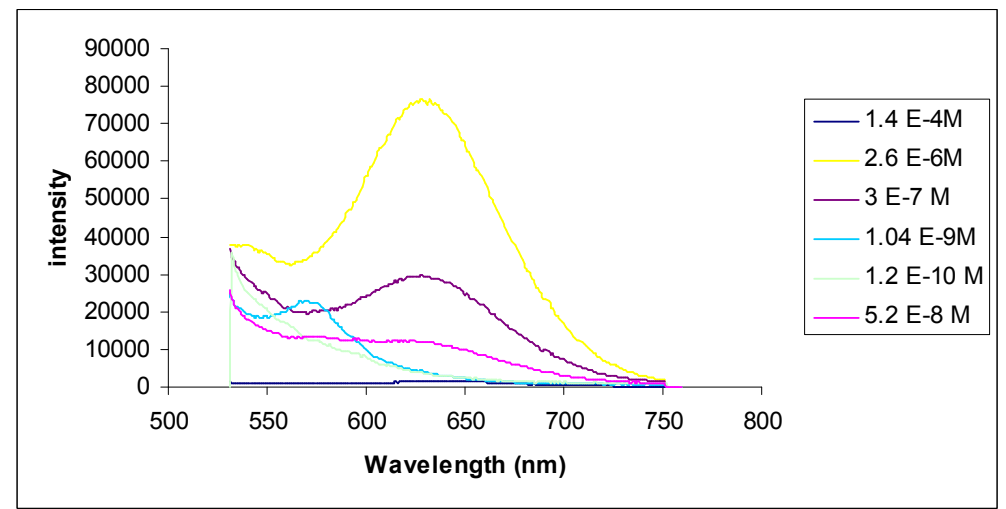




\section{Copies of ${ }^{1} \mathrm{H}$ and ${ }^{13} \mathrm{C}$ NMR spectra}

${ }^{1} \mathrm{H}$ and ${ }^{13} \mathrm{C}$ NMR spectra (400 and $100 \mathrm{MHz}$ respectively, $\mathrm{CDCl}_{3}$ ) of $\mathbf{3}$

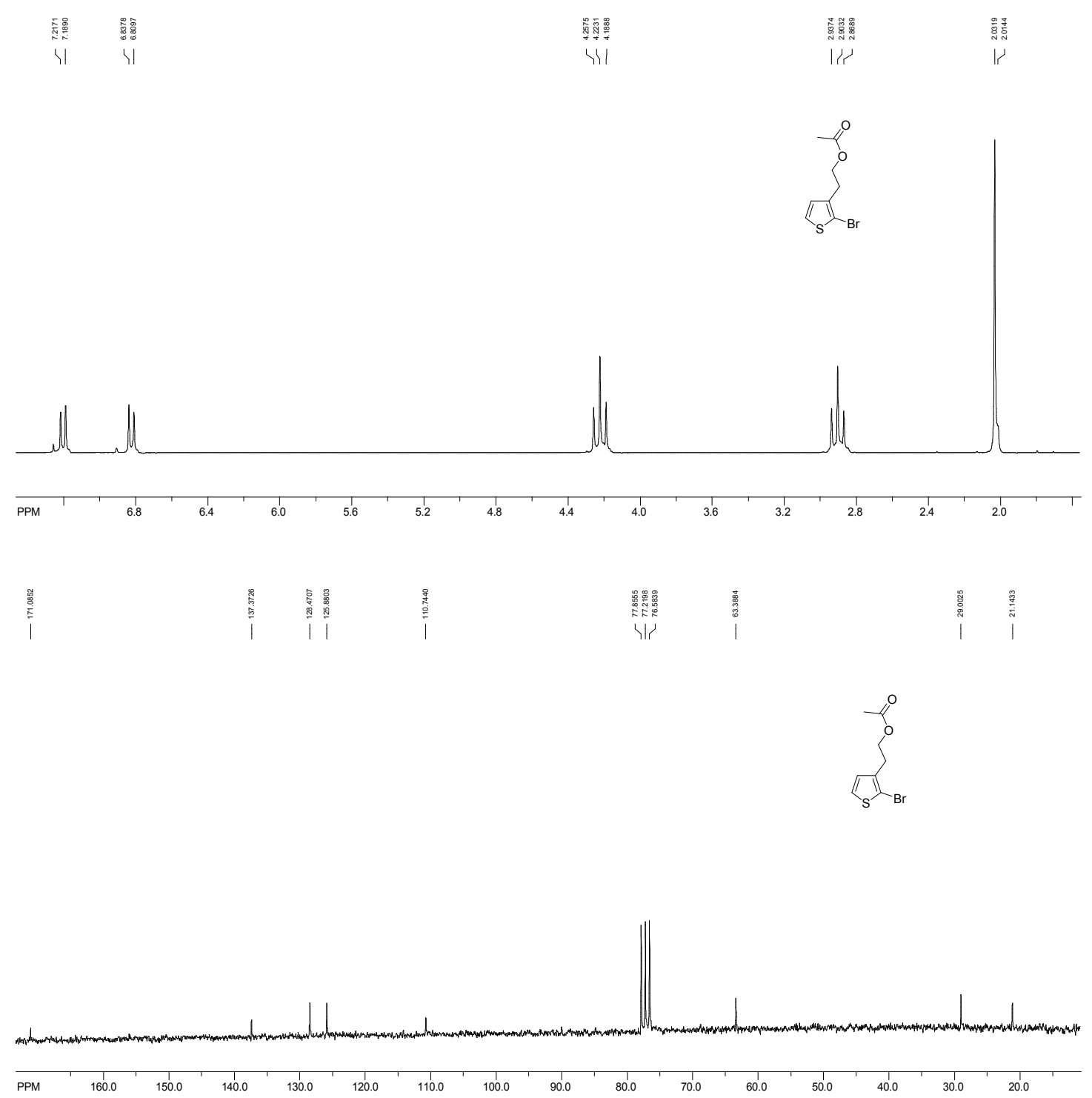


${ }^{1} \mathrm{H}$ and Dept $135 \mathrm{NMR}$ spectra (400 and $100 \mathrm{MHz}$ respectively, $\mathrm{CDCl}_{3}$ ) of 6
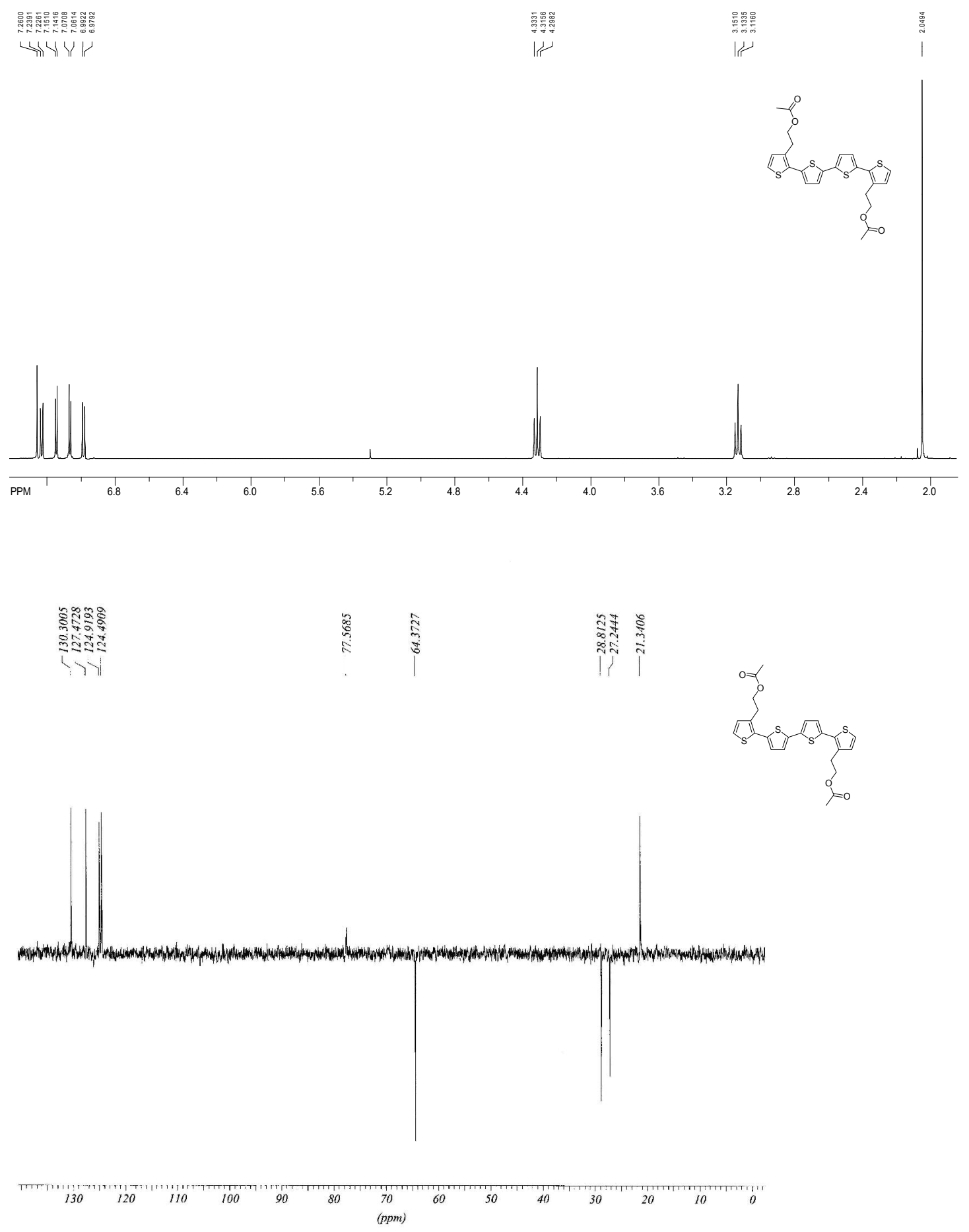
${ }^{1} \mathrm{H}$ and ${ }^{13} \mathrm{C}$ NMR spectra (400 and $100 \mathrm{MHz}$ respectively, $\mathrm{CDCl}_{3}$ ) of 7

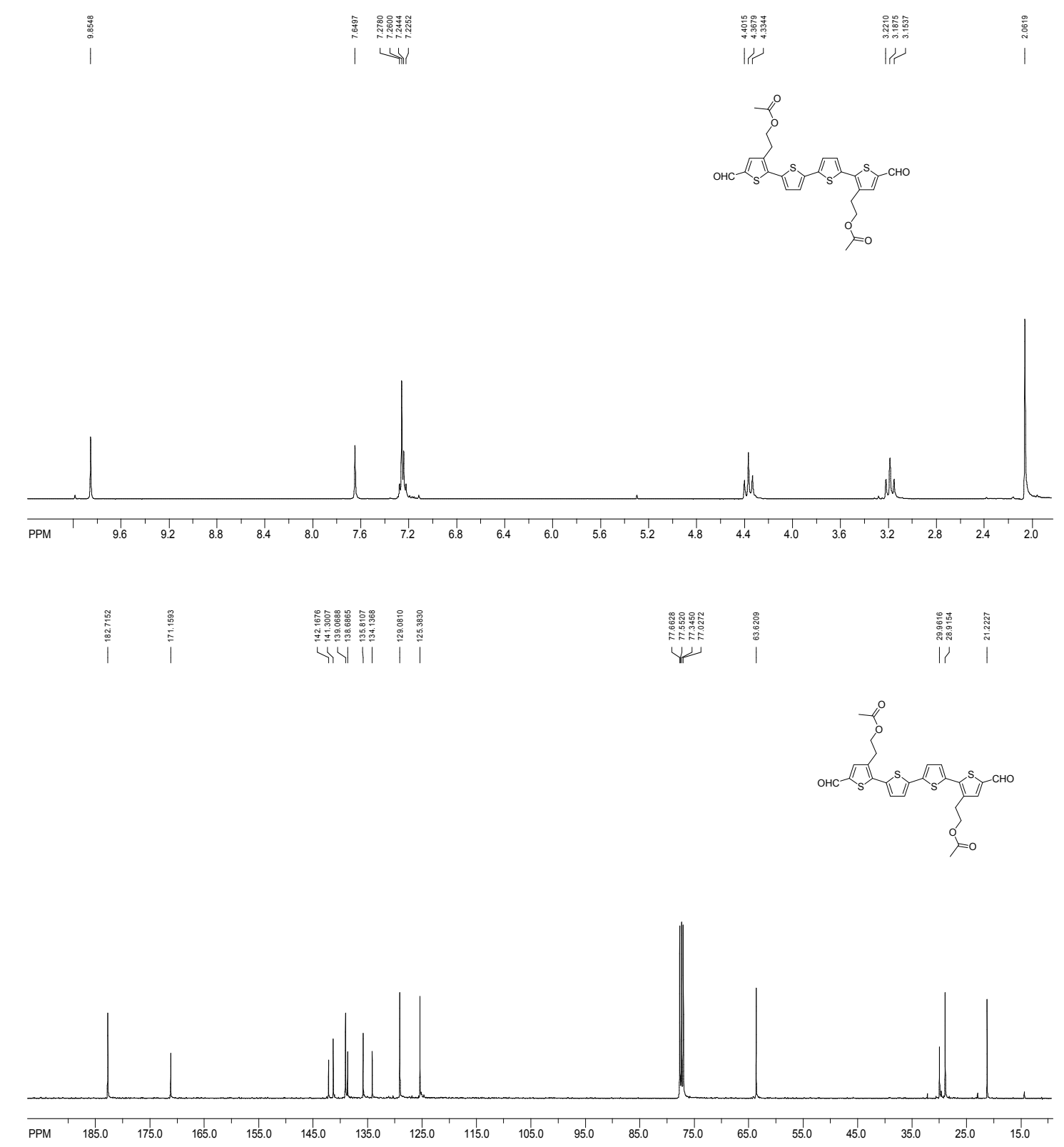


${ }^{1} \mathrm{H}$ and ${ }^{13} \mathrm{C}$ NMR spectra (400 and $100 \mathrm{MHz}$ respectively, $\mathrm{CDCl}_{3}$ ) of 8

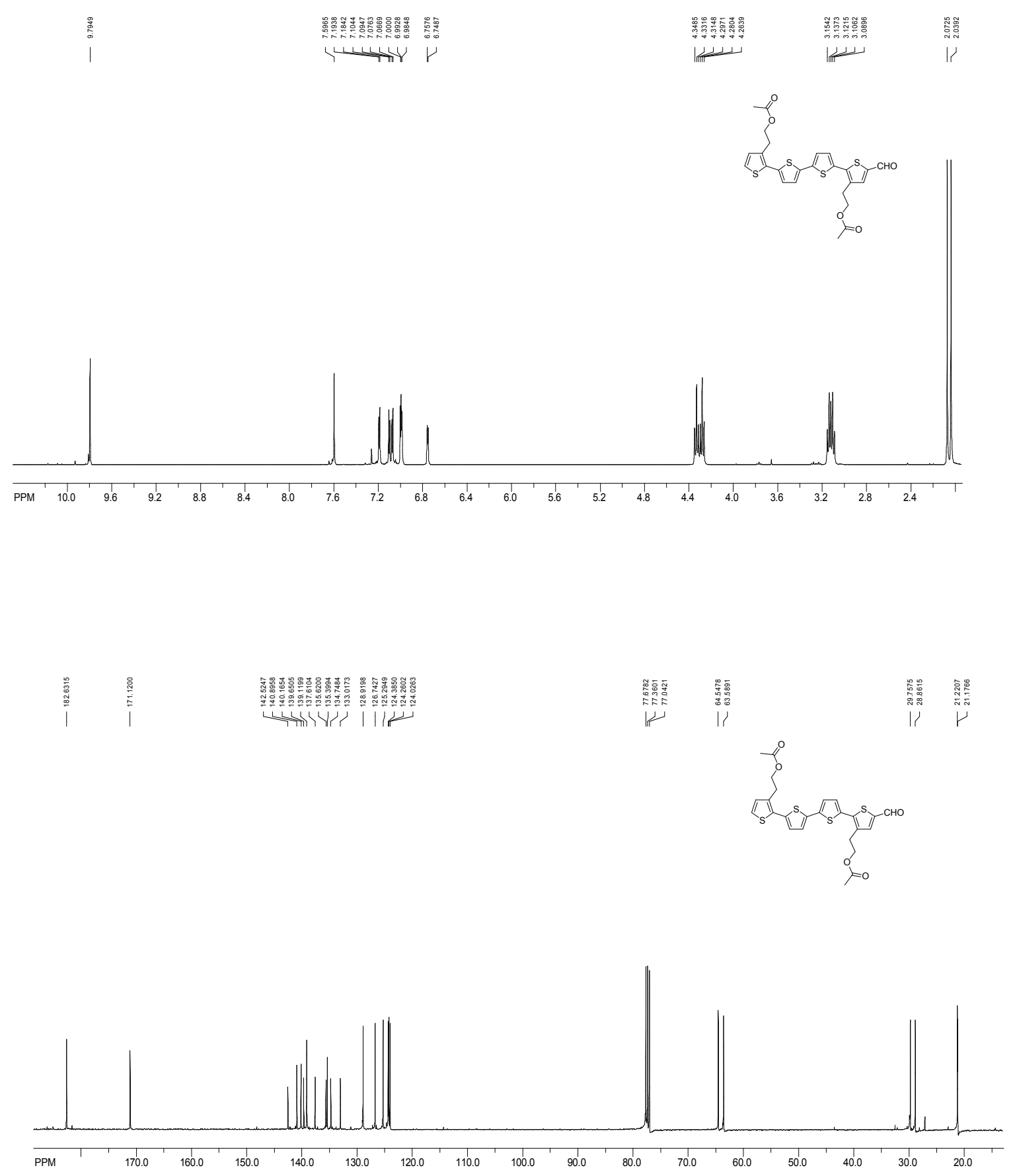


${ }^{1} \mathrm{H}$ and ${ }^{13} \mathrm{C}$ NMR spectra (400 and $100 \mathrm{MHz}$ respectively, $\mathrm{CDCl}_{3}$ ) of 9

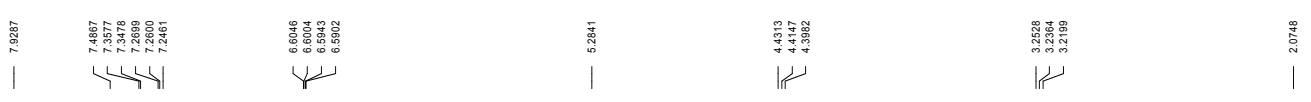
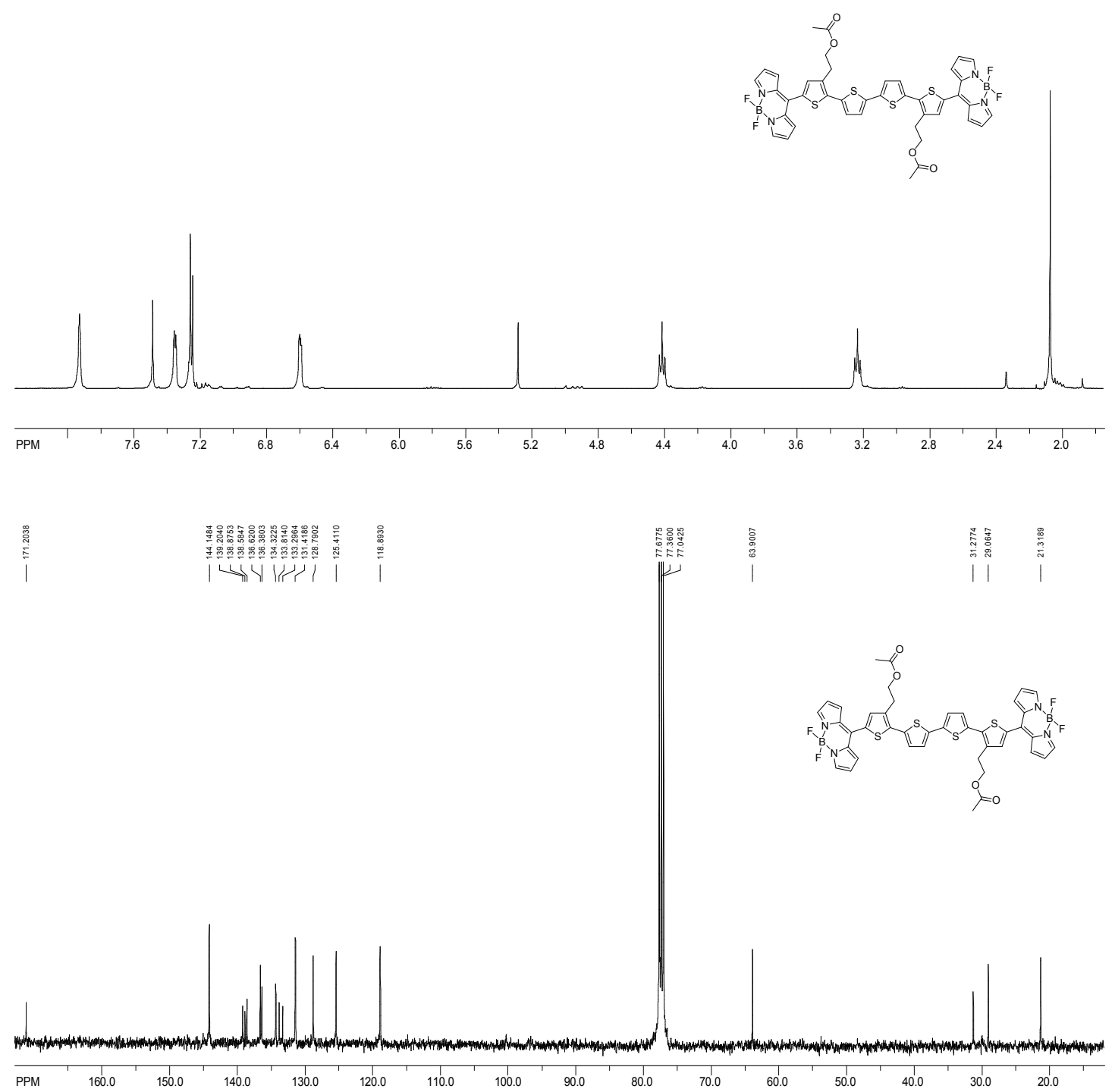
${ }^{1} \mathrm{H}$ and ${ }^{13} \mathrm{C}$ NMR spectra (400 and $100 \mathrm{MHz}$ respectively, $\mathrm{CDCl}_{3}$ ) of $\mathbf{1 0}$
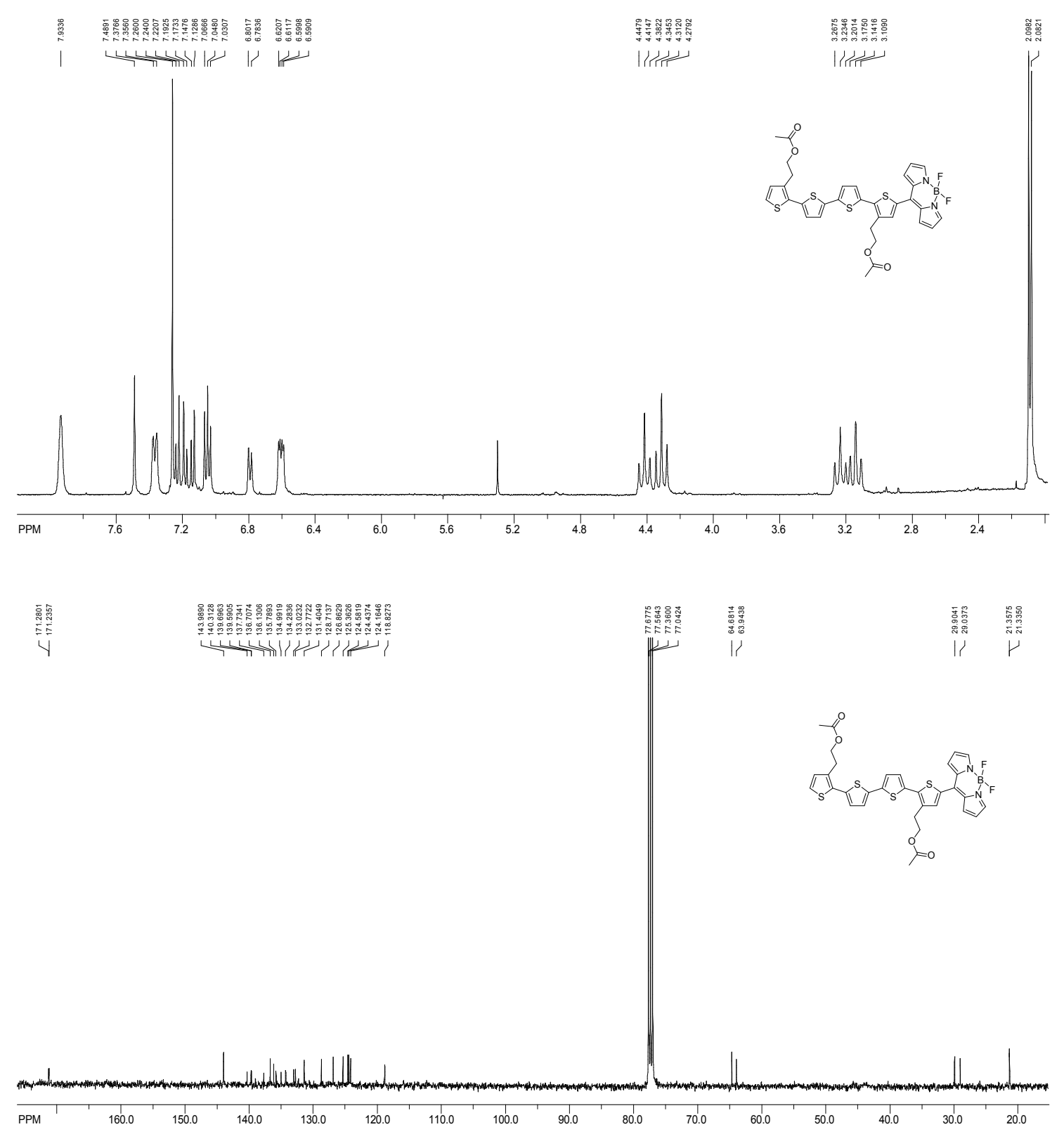